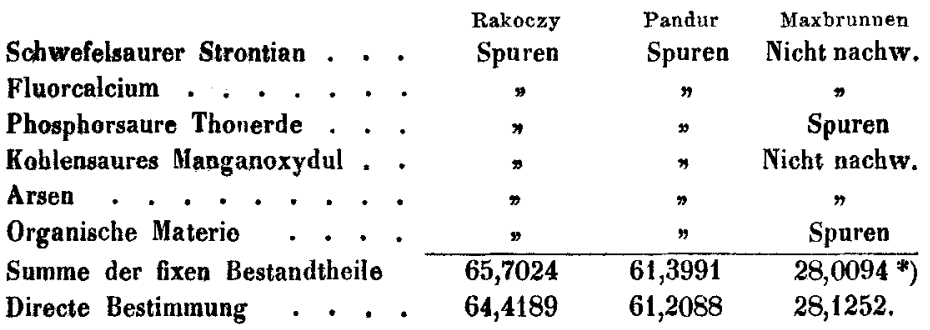

Gesammtvolum der freien und halbgebundenen Kohlensäure bei der Quellentemperatur und $760^{\mathrm{mm}} \mathrm{B}$.

In 100 Liter Wasser Rakoczy Pandur Maxbrumnen In 1 Pfund $=32$ Cubikzoll Wasser $41,77 \mathrm{CZ}$. $48,17 \mathrm{CZ} . \quad 41,85 \mathrm{CZ}$.

Die im Wasser aufsteigenden Gase enthalten in 100 Volumina's :

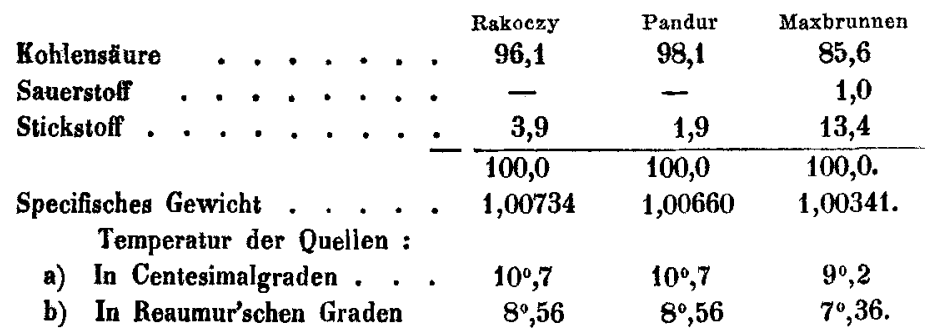

\title{
Untersuchung einiger Pflanzenproducte aus Indien;
}

$$
\text { von J. Stenhouse. }
$$

(Gelesen vor der Royal Society zu London am 6." December 1855.)

Der Gefälligkeit meines geehrten Freundes Dr. Royle verdanke ich es, dals ich aus der reichhaltigen Sammlung des Ostindia-Hauses diejenigen Pflanzenproducte auswählen konnte, deren Untersuchung von besonderem Interesse zu

*) In der Summe ist das Ammoniak nicht mitgezählt. 
sein schien. Während der letzten zwölf Monate beschäftigte ich mich hauptsächlich mit drei dieser Producte, und die Resultate dieser Untersuchungen theile ich hier mit, und werde später die Ergebnisse meiner weiteren Studien in dieser Richtung nachfolgen lassen.

\section{Datisca cannabina.}

Die erste der drei von mir untersuchten Substanzen bestand aus einer Quantitäl der Wurzeln von Datisca cannabina aus Lahore, wo diese Pflanze angewendet wird um Seide dauerhaft gelb zu färben. Die in etwa 6 bis 8 Zoll lange Stücke zerschnittenen Wurzeln waren $\frac{1}{2}$ bis $\frac{3}{4}$ Zoll dick. Sie waren tief gelb gefärbt.

Die Blätter und dünneren Zweige der Datisca cannabina aus der Levante sind lange im südlichen Frankreich zu gleichem $Z$ wecke angewendet worden. Ein Decoct dieser Blätter wurde 1816 durch Braconnot untersucht, welcher darin einen krystallisirbaren Bestandtheil entdeckte, welchen er Datiscin nannte*). Bracon n ot konnte damals diesen Körper nicht der Elementaranalyse unterwerfen, aber seine Angaben über das Aussehen und die Eigenschaften desselben sind sehr genau. Die von Braconnot erhaltenen Resultate wurden indefs so sehr vergessen, dafs während vieler Jahre in den meisten der umfassenderen Lehrbüchern der Chemie Datiscin als gleichbedeutend mit Inulin sich angegeben findet. So wird in Brande's Chemie, Bd. II, S. 1168 angegeben, "Inulin sei häufig auch anders benannt worden, wie z. B. Dahlin, Datiscin u. a." In Löwig's Chemie der organischen Verbindungen, Bd. I, S. 359 findet man dieselbe irrige Angabe wiederholt, wo sich unter den Synonymen für Inulin auch Dahlin und Datiscin angegeben finden.

*) Ann. chim. phys. [2] III, 277. 
Die zerkleinerten Wurzeln wurden in einem Mohr'schen Extractionsapparat während längerer Zeit mit Holzgeist ausgezogen. Der so erhaltene Auszug war dunkelbraun gefärbt; er wurde durch Abdestilliren eines Theils des Holzgeists concentrirt. Die in der Retorte zuriackgebliebene braune syrupartige Flüssigkeit wurde in offenen Gefälsen stehen gelassen und schied nach einiger Zeit eine harzartige Substanz aus, in welcher nur Spuren von Krystallen wahrzunehmen waren. Als indefs diese syrupartige Flüssigkeit mit ihrem halben Volum an heilsem Wasser behandelt wurde, schied sich der gröfsere Theil der harzartigen Substanz rasch ab, und die von der letzteren abgegossene Flüssigkeit gab bei langsamem Verdunsten eine beträchtliche Menge eines unvollkommen krystallisirten, wie Traubenzucker aussehenden Körpers.

Dieser Körper ist unreines Datiscin, welches noch eine grolse Menge harzartiger Substanz zurückhält; auf dem Gehalt an letzterer beruht die dunkelbraune Färbung. Das Datiscin läfst sich in verschiedener Weise reinigen, wobei man namentlich benutzen kann, dals die harzartige Substanz in Alkohol und in Aether leichter löslich ist, als das Datiscin. Durch wiederholtes Umkrystallisiren aus einem dieser beiden Lösungsmittel kann man das Datiscin fast vollkommen farblos erhalten, indem die Verunreinigungen in den Mutterlaugen bleiben. Folgendes ist das Verfahren, welches ich im Ganzen als das zweckentsprechendste gefunden habe. Das rohe, noch feuchte Datiscin wird zwischen Fliefspapier stark ansgeprefst, dann in Alkohol aufgelöst und die Lösung mit Wasser versetzt, wodurch harzartige Substanz niedergeschlagen wird. Die verdünnte alkoholische Lösung scheidet nach einiger Zeit das Datiscin in reinerem Zustande ab. Durch offteres Wiederholen dieses Verfahrens lälst sich das Datiscin vollkommen rein erhalten. Um jede Spur Gerbsäure, welche 
zugegen sein möchte, zu entfernen, versetzte ich zu wiederholten Malen die alkoholische Lösung des Datiscins mit einer concentrirten Leimlösung, und schied nach dem Filtriren das Datiscin in der bereits angegebenen Weise aus. Ich konnte indessen nicht wahrnehmen, dals die Krystalle des Datiscins, wenn Leimlösung zur Reinigung desselben angewendet worden war, sich irgend von denjenigen unterschieden hätten, welche ohne Anwendung dieses Reinigungsmittels erhalten worden waren.

Eigenschaften des Datiscins. - Das ganz reine Datiscin ist vollkommen farblos, aber wenn es nicht mit der gröfsten Sorgfalt gereinigt wurde, ist es gewöhnlich mehr oder weniger gelblich gefärbt. Es ist leicht löslich in Alkohol, selbst in kaltem; in siedendem Alkohol löst es sich fast nach jedem Verhältnifs. Bei dem freiwilligen Verdunsten der alkoholischen Lösung scheiden sich kleine seideartige, zu Gruppen verwachsene Nadeln aus. In kaltem Wasser löst es sich nur wenig, in siedendem etwas reichlicher, und bei dem Erkalten der Ietzteren Lösung scheidet es sich in glänzenden Blättchen ab. In Aether löst sich das Datiscin nur spärlich, aber eine solche Lösung giebt bei sehr langsamem Verdunsten des Aethers gröfsere Krystalle von Datiscin, als aus irgend einer anderen Lösung erhalten werden konnten. Bei Zusatz von Wasser zu einer alkoholischen Lösung von Datiscin bildet sich, aufser wenn die Lösung sehr concentrirt war, nicht sofort ein Niederschlag, aber bei längerem Stehen der Flüssigkeit scheidet sich das Datiscin sehr rein in Form blafsgelber Krystalle aus. Das so dargestellte Datiscin ergab noch einen sehr unerheblichen Gehalt an unverbrennlichen Bestandtheilen. - Auf etwa $180^{\circ} \mathrm{C}$. erhitzt schmilzl das Datiscin, und bei weiterer Steigerung der Temperatur verbrennt es unter Ausstofsung des Geruchs nach gebranntem Zucker und Hinterlassung eines voluminösen kohligen Rück- 
stands. Wird Datiscin in einem abgeschlossenen Raume unter Ueberleiten eines langsamen Stroms trockener Luft erhitzt, so sublimirt eine geringe Menge einer krystallinischen Substanz. - Datiscin und seine Lösungen schmecken stark bilter; obgleich es sich gegen Reagenspapiere indifferent verhält, glaube ich es doch als einen Körper von schwach sauren Eigenschaften betrachten zu können. - Es löst sich in den wässerigen Lösungen der fixen Alkalien und des Ammoniaks, auch in Kalk - und Barytwasser. Diese Lösungen besitzen eine tief gelbe Farbe, welche bei Zusalz einer Säure (selbst von Essigsäure) unter Ausscheidung des Datiscins verschwindet. Wenn die alkalischen Lösungen nicht allzu concentrirt sind und noch heifs mit einer Säure neutralisirt werden, so scheidet sich das Datiscin bei dem Erkalten in kleinen Krystallen $a b$. Die wässerige Lösung des Datiscins wird durch neutrales und durch basisches essigsaures Bleioxyd und durch Zinnchlorid gefällt; die Niederschläge sind hellgelb gefärbı. Kupfersalze bringen grünliche, Eisenoxydsalze dunkel-bräunlichgrüne Niederschläge hervor; das zu diesen Reactionen verwendete Datiscin war mittelst Leimlösung gereinigt worden. Die durch die Bleioxydsalze hervorgebrachten Niederschläge sind so gallertartig, dafs sie nicht ausgewaschen werden konnten, und ich konnte sie defshalb nicht zur Feststellung des Aequivalentgewichts des Datiscins benutzen.

Einwirkung verdünnter Schwefelsäure auf Datiscin. Wird eine wässerige Lösung von Datiscin mit einer kleinen Menge Schwefelsäure versetzt und die Flüssigkeit gekocht, so trübt sich dieselbe nach wenigen Minuten und eine krystallinische Substanz scheidet sich aus. Diese wurde abfiltrirt, und das klare Filtrat zeigte nach dem Zusatz von Kali bis zu stark alkalischer Reaction das Vermögen, bei $100^{\circ}$ Kupferoxyd zu Oxydul zu reduciren. Wird aus einem anderen Theile des Filtrats die Schwefelsäure millelst kohlensauren 
Bleioxyds oder kohlensauren Baryts entfernt, so wird die Flüssigkeit süfsschmeckend und giebt bei dem Verdunsten einen Syrup, welcher nach längerem Stehen zu einer halbkrystallinischen, dem Honig sehr ähnlichen Masse wird. Es geht hieraus hervor, dafs das Datiscin, analog wie das Salicin und ähnliche Substanzen, zu den s. g. Glucosiden gehört, und eine gepaarte Verbindung von Zucker und einem anderen Körper ist, welchen ich als Datiscetin bezeichnen will.

Datiscetin. - Das Datiscetin gleicht hinsichtlich des äufseren Ansehens dem Datiscin, aber bei genauerer Untersuchung ergiebt es doch hinsichtlich der Zusammensetzung und der Eigenschaften wesentliche Verschiedenheiten. Datiscetin, dargestellt durch Kochen einer Lösung von reinem Datiscin mit verdünnter Schwefelsäure, schlägt sich in Form von Nadeln nieder, welche fast farblos sind. Es löst sich leicht in Alkohol und scheidet sich aus der heifsen alkoholischen Lösung bei dem Abkühlen derselben zum gröfseren Theil krystallinisch aus. Es ist fast unlöslich in Wasser, und wird dem entsprechend aus seiner alkoholischen Lösung auf Zusatz von Wasser grölstentheils niedergeschlagen. Es löst sich fast nach jedem Verhältnifs in Aether, und scheidet sich bei dem Verdunsten dieses Lösungsmittels in Nadeln aus. Dieses Verhalten des Datiscetins ermöglicht es, dasselbe in reinem Zustande gewinnen zu lassen, selbst wenn das zu seiner Bereitung verwendete Datiscin sehr unrein war. Dic Flüssigkeiten, aus welchen sich Datiscin krystallinisch ausgeschieden hatte, können noch in folgender Weise zur Darstellung von Datiscetin verwendet werden. Diese Flüssigkeiten werden zuerst mit basisch-essigsaurem Bleioxyd behandelt, der Niederschlag auf einem Filter gesammelt und ausgewaschen, dann in Wasser zertheilt und mittelst Schwefelwasserstoffs zersetzt. Die Flüssigkeit wird nach dem Abfiltriren des Schwefelbleis concentrirt und mit verdünnter Schwefelsäure 
gekocht. Das auf diese Art dargestellte Datiscetin kann leicht von harzartiger Substanz gereinigt werden, da die letztere durch die Schwefelsäure schon vor der Bildung des Datiscetins abgeschieden wird und sich an den Boden des Kolbens anlegt. Durch Auflösen des Datiscetins in Alkohol und Fällen mit Wasser wird es nahezu rein erhalten.

Eigenschaften des Datiscetins. - Das Datiscetin ist geschmacklos. Bei dem Erhitzen schmilzt es wie das Datiscin, aber erst bei höherer Temperatur; bei dem Erkalten erstarrt es krystallinisch. Bei sehr vorsichtigem Erhitzen kann das Datiscetin theilweise zu Krystallen sublimirt werden, wobei es indefs doch einige Veränderung zu erleiden scheint, denn das Sublimat hat nach dem Unkrystallisiren aus Aether einen süfsen Geschmack. Bei dem Verbrennen entwickelt das Datiscetin nicht den Geruch nach gebranntem Zucker. Es löst sich, wie das Datiscin, in alkalischen Flüssigkeiten, und wird aus diesen Lösungen durch Säuren wieder gefällt. Bei Mischung der alkoholischen Lösungen von essigsaurem Bleioxyd und Datiscetin bildet sich ein schön gefärbter, tief gelber Niederschlag, welcher sich mit Alkohol und mit Wasser gut auswaschen läfst, und der Analyse unterworfen werden konnte.

I. 0,4555 Grm. gaben $0,2060 \mathrm{PbO}$.

$0,2655 "$ " $0,3515 \mathrm{CO}_{2}$ und $0,0405 \mathrm{HO}$.

II. $0,4310, n \quad 0,1945 \mathrm{PbO}$.

III. (Von einer anderen Darstellung) :

0,4170 Grm. gaben 0,1907 Pb0.

$0,3170 " \quad 0,4195 \mathrm{CO}_{2}$ und $0,0460 \mathrm{HO}$.

Diese Resultate führen zu der Formel $\mathrm{C}_{30} \mathrm{H}_{8} \mathrm{O}_{10}+2 \mathrm{PbO}$ :

\begin{tabular}{rrrrrrr} 
& \multicolumn{2}{c}{ berechnet } & & \multicolumn{3}{c}{ gefunden } \\
\cline { 2 - 3 } $\mathrm{C}_{30}$ & 180,0 & 36,63 & & \multicolumn{1}{c}{ I. } & II. & III. \\
$\mathrm{H}_{8}$ & 8,0 & 1,63 & & 1,69 & - & 36,09 \\
$\mathrm{O}_{10}$ & 80,0 & 16,28 & & $-1,61$ \\
$2 \mathrm{Pb0}$ & 223,4 & 45,46 & 45,22 & 45,13 & 45,73 \\
\hline & 491,4 & $100,00$. & & &
\end{tabular}


Bei der Analyse des freien Datiscetins erhielt ich folgende Zahlen (die Substanz war stets bei $100^{\circ}$ in einem Strom trockener Luft getrocknet, und wurde in einem Gasofen verbrannt) :

Datiscetin, aus Alkohol umkrystallisirt :

I. $0,3970 \mathrm{Grm}$. gaben $0,9155 \mathrm{CO}_{2}$ und $0,1280 \mathrm{HO}$.

II. $0,4455, \quad 1,0275, \quad, 0,1485$,

Datiscetin, aus der alkoholischen Lösung mittelst Wasser gefällt :

III. 0,2045 Grm. gaben 0,4715 $\mathrm{CO}_{2}$ und $0,0665 \mathrm{HO}$.

IV. 0,3680 \# $0,8505 \%$ 0,1245

Die aus diesen Zahlen sich berechnende procentische Zusammensetzung entspricht sehr nahe derjenigen, welche von der aus der Analyse des Bleisalzes abgeleiteten Formel gefordert wird.

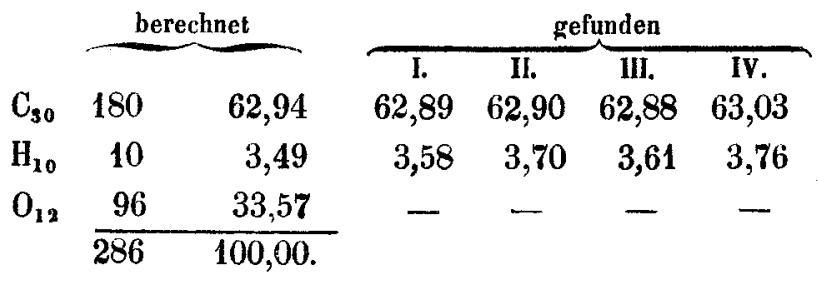

Bei der Analyse des Datiscins erhielt ich folgende Resultate :

Datiscin, aus Alkohol krystallisirt :

I. $0,3890 \mathrm{Grm}$. gaben $0,7815 \mathrm{CO}_{2}$ und $0,1780 \mathrm{HO}$.

II. $0,3395 \Rightarrow \quad 0,6770$ \# 0,1585 ,

Datiscin, aus Alkohol krystallisirt, von einer anderen Bereilung :

III. $0,2785 \mathrm{Grm}$, gaben $0,5595 \mathrm{CO}_{2}$ und $0,1315 \mathrm{HO}$.

IV. 0,2905 " 0,5830 \# 0,1385 "

Datiscin, mit Anwendung von Leimlösung dargestellt, aus Alkohol krystallisirt :

V 0,4145 Grm. gaben $0,8355 \mathrm{CO}_{2}$ und $0,1925 \mathrm{HO}$. 
Datiscin, aus der alkoholischen Lösung mittelst Wasser ausgefällt :

VI. 0,1580 Grm. gaben 0,3135 $\mathrm{CO}_{2}$ und $0,0770 \mathrm{HO}$.

VII. 0,1980 " $0,3955, \quad 0,0925$,

Datiscin, aus der Lösung in Aetzkali durch Essigsäure gefällt :

VIII. $0,2985 \mathrm{Grm}$. gaben $0,5940 \mathrm{CO}_{2}$ und 0,1375 $\mathrm{HO}$.

Die bei den Analysen I und II angewendete Substanz enthielt 0,3 bis $0,4 \mathrm{pC}$. Asche, die bei III und IV angewendete 0,2 bis $0,25 \mathrm{pC}$, und die bei $\mathrm{V}$ angewendete $0,125 \mathrm{pC}$. Auf den Aschengehalt ist in der unten folgenden Berechnung der procentischen Zusammensetzung keine Rücksicht genommen. Das für die Analysen VI, VII und VIIl verwendete Datiscin enthielt keine bestimmbare Menge Asche.

I. II. III. IV. V. VI. VII. VIII. $\begin{array}{lllllllll}\text { C } & 54,79 & 54,38 & 54,79 & 54,73 & 54,97 & \mathbf{5 4 , 1 1} & \mathbf{5 4 , 4 8} & 54,27\end{array}$

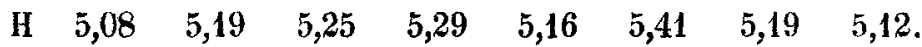

Es ist schwierig, eine Formel aufzustellen, welcher die hier gefundenen Zahlen genügend entsprechen. Bei Berücksichtigung der Spaltung des Datiscins in Zucker und Datiscetin hat für das erstere die Formel $\mathrm{C}_{42} \mathrm{H}_{22} \mathrm{O}_{24}$ eine gewisse Wahrscheinlichkeit.

$$
\underset{\text { Datiscetin }}{\mathrm{C}_{30} \mathrm{H}_{10} \mathrm{O}_{12}}+\underset{\text { Zucker }}{\mathrm{C}_{12} \mathrm{H}_{12} \mathrm{O}_{12}}=\underset{\text { Datiscin }}{\mathrm{C}_{42} \mathrm{H}_{22} \mathrm{O}_{24}} \text {; }
$$

nach dieser Formel berechnet sich die procentische Zusammensetzung :

$$
\begin{array}{rrr}
\mathrm{C}_{42} & 252 & 54,08 \\
\mathrm{H}_{22} & 22 & 4,72 \\
\mathrm{O}_{24} & 192 & 41,20 .
\end{array}
$$

Wenn diese Formel $\mathrm{C}_{42} \mathrm{H}_{22} \mathrm{O}_{24}$ die richtige ist, so geht die Spaltung des Datiscins ähnlich vor sich wie die des Salicins, welches bei dem Erhitzen mit einer verdb̈nnten Säure zerfialll entsprechend der Gleichung: 


$$
\underset{\text { Salicin }}{\mathrm{C}_{26} \mathrm{H}_{18} \mathrm{O}_{14}}=\underset{\text { Zucker }}{\mathrm{C}_{12} \mathrm{H}_{12} \mathrm{O}_{12}}+\underset{\text { Saliretin }}{\mathrm{C}_{14} \mathrm{H}_{8} \mathrm{O}_{2}} \text {, }
$$

während die Spaltung bei Einwirkung von Emulsin folgende ist :

$$
\underset{\text { Salicin }}{\mathrm{C}_{26} \mathrm{H}_{18} \mathrm{O}_{14}}=\underset{\text { Zucker }}{\mathrm{C}_{12} \mathrm{H}_{12} \mathrm{O}_{12}}+\underset{\text { Saligenin. }}{\mathrm{C}_{14} \mathrm{H}_{8} \mathrm{O}_{4}}-2 \mathrm{HO}
$$

Die oben gegebene Formel für das Datiscin findet ihre Bestätigung in den Bestimmungen, wie viel Zucker aus einer bekannten Menge Datiscin bei Behandlung mit verdünnter Schwefelsäure entsteht. Der Zucker wurde nach Fehling's Methode mittelst einer titrirten Lösung ron schwefelsaurem Kupferoxyd bestimmt. Vier auf diese Art angestellten Versuche gaben für die aus 100 Theilen Datiscin sich bildende Menge Zucker 41,6, 39,5, 39,3 und 37,8 Th., während sich nach der Formel $\mathrm{C}_{42} \mathrm{H}_{22} \mathrm{O}_{24} 38,6$ Gewic'ststheile bilden sollten.

Ich versuchte auch, diese Formel durch die Bestimmung der Quantität Datiscetin zu controlliren, welche sich bei der Behandlung einer gewogenen Menge Datiscin mit verdünnter Schwefelsäure bildet. Die bei verschiedenen Versuchen gefundenen Resultate stimmten indefs nicht unter einander überein, und ergaben sämmtlich viel niedrigere Zahlen, als man nach der obigen Formel erwarten sollte. Diefs kann indefs nicht wundern, da das Datiscetin, wenn auch schwerlöslich in Wasser, doch nicht unlöslich in demselben ist.

Verdünnte Salzsäure wirkt, wie verdünnte Schwefelsäure, auf das Datiscin unter Bildung von Zucker und Datiscetin ein. Selbst schon bei mehrstündigem Kochen einer wässerigen Lösung von Datiscin bilden sich Spuren von Zucker, zum Beweis, dals die Spaltung einer kleinen Menge Datiscin bereits unter diesen Umständen vor sich geht. Durch frühere Versuche und durch die Analyse VIII (S. 174) war festgestellt, dals das Datiscin in kalter Kalilauge ohne Zersetzung löslich ist. Wird es indessen mit concentrirter Kalilauge während einiger Zeit gekocht, so tritt Zersetzung ein und 
der auf Zusatz einer Säure sich bildende Niederschlag hat nun alle Eigenschaften des Datiscetins. In dieser Beziehung verhält sich also das Datiscin wie die Gerbsäure und andere Glucoside, welche in ähnlicher Weise bei Einwirkung von Säuren oder Alkalien gespalten werden; Galläpfel-Gerbsäure wird z. B. durch Säuren und Alkalien zu Gallussäure und Zucker gespalten. Wird eine Lösung von Datiscin in Berührung mit Hefe längere Zeit an einem warmem Platze stehen gelassen, so zeigt sich keine Gährung, und auch bei Einwirkung von Emulsin liefs sich keine Abscheidung von Datiscelin wahrnehmen.

Einwirkung der Salpetersäure auf Datiscin und Datiscetin. - Wird Datiscetin in der Kälte mit Salpetersäure von gewöhnlicher Concentration behandelt, so trilt eine lebhafte Einwirkung ein, braune Dämpfe entwickeln sich, und eine harzarlige Substanz wird gebildel. Die bei der Einwirkung selbst frei werdende Wärme ist hinreichend, die erstere so weit fortdauern zu lassen, dafs die harzartige Substanz wieder gelöst wird und eine dunkelrothe Flüssigkeit entsteht, welche nach dem Kochen gelb wird und nach vorsichtigem Abdampfen bei dem Abkühlen Krystalle von Pikrinsalpetersäure giebt., Wenn die Einwirkung nicht allzu weit gegangen ist, entsteht keine Oxalsäure, und die dabei erbaltene Pikrinsalpetersäure ist sehr rein und bildet grofse Krystalle von blafsgelber Farbe. Die folgenden Versuche zeigen, dafs diese Krystalle wirklich Pikrinsalpetersäure sind. Bei Behandlung mit einer concentrirten Lösung von unterchlorigsaurem Kalk in der Kälte trilt sogleich der characteristische Geruch des Chlorpikrins auf. Bei Zusatz von Kalilösung zu einem anderen Theil der Säure scheiden sich Krystalle von reinem pikrinsalpetersaurem Kali aus, welche 18,04 pC. Kali ergaben, während sich nach der Formel des pikrinsalpetersauren Kalis 17,66 pC. berechnen. - Datiscin giebt bei 
gleicher Behandlung mit Salpetersäure Pikrinsalpetersäure und Oxalsäure.

Die Bildung von Pikrinsalpetersäure bei Einwirkung starker Salpetersäure auf Datiscin und Datiscetin machte es sehr wahrscheinlich, dafs bei Anwendung verdünnterer Säure andere, auf geringerer Oxydation beruhende Zersetzungsproducte erhalten werden möchten. Datiscetin wurde defshalb mit verdünnterer Salpetersäure (1 Theil gewöhnlicher Säure auf 10 Theile Wasser) gekocht; die Krystalle des Datiscetins lösten sich bald zu einer gelben Flüssigkeit, welche bei Behandlung mit einer Lösung von Eisenchlorid sich blutrolh färbte. Bei dem Erkalten der ursprünglichen gelben Lösung schieden sich blafsgelbe Krystalle ab. Diese waren leichtlöslich in heifsem Wasser und schieden sich bei dem Abkühlen wieder aus; sie lösten sich auch leicht in Alkohol und in Aether, und die alkoholische Lösung gab bei langsamem Verdunsten fast farblose, ziemlich grofse, seideartig glänzende Krystalle. Bei dem Behandeln mit unterchlorigsaurem Kalk entwickelten sie nicht den Geruch nach Chlorpikrin, wohl aber bei schwachem Erwärmen. Bei vorsichtigem Erhitzen der Krystalle zwischen zwei Uhrgläsern konnte ein Theil zu farblosen Nadeln sublimirt werden. Bei dem Erhitzen dieser Säure mit einer zur vollständigen Lösung unzureichenden Menge Wasser schmolz sie und krystallisirte bei dem Abkühlen wieder. Diese Eigenschaften stimmen mit denen der Nitrosalicylsäure überein. Um über die Identität beider Subslanzen urtheilen zu können, wurden die folgenden Salze dargestellt. Durch Sättigen einer Lösung der Säure mit kohlensaurem Baryt wurde ein Barytsalz in gelben Krystallen erhalten, und aus seiner Lösung fällte Ammoniak ein anderes gelb gefärbtes Barytsalz. Auch ein krystallinisches Bleisalz konnte mittelst der Lösung der Säure erhalten werden. Das Ammoniaksalz wurde durch Sättigen der Lösung 
der Säure mit Ammoniak und Krystallisirenlassen im leeren Raume in orangefarbenen Nadeln erhalten. Mittelst des letzteren Salzes wurde durch doppelte Zersetzung die Silberverbindung erhalten, welche, wie auch alle andern Salze, in ihren Eigenschaften aufs genaueste mit der von Marchand beschriebenen entsprechenden Verbindung der Nitrosalicylsäure übereinstimmte. Bei der Analyse gaben 0,1695 Grm. des Silbersalzes 0,0630 Silber, entsprechend 37,17 pC.; in dem nitrosalicylsauren Silber sind 37,24 pC. enthalten. Wird Datiscin mit verdünnter Salpetersäure in der Kälte zusammenstehen gelassen, so löst es sich allmälig auf, und die Flüssigkeit nimmt eine gelbe Farbe an. Bei dem Verdunsten der Lösung im leeren Raume blieb ein Gemenge von Oxalsảure und Pikrinsalpetersäure zurück.

Einwirkung von Kali auf Datiscin und Datiscetin. - Ich habe bereits oben angegeben, dais Datiscin und Datiscetin sich in wässerigen ätzenden Alkalien ohne Zersetzung lösen, und dafs das Datiscin bei längerem Kochen mit Kalilauge unter Bildung von Datiscelin zersetzt wird. Es blieb noch übrig, die Einwirkung von schmelzendem Kalihydrat zu untersuchen. Wird Datiscelin in kleinen Portionen allmälig zu schmelzendem Kalihydrat gesetzt, so färbt es sich tief-orangefarben und löst sich dann unter Entwickelung von Wasserstoffgas. Nach dem Aufhören der Wasserstoflentwicklung wurde die Masse in Wasser gelöst und mit Salzsäure übersättigt. Eine zum Theil harzige Substanz schied sich ab, welche bei der Sublithation farblose lange Krystalle gab, die der Benzoësärre glichen. Die wässerige Lösung derselben zeigte auf Zusatz von Eisenchlorid die tief violette, auf Zusatz von Salzsäure verschwindende Färbung, welche für die Salicylsäure characteristisch ist.

Einwirkung der Chromsäure. - Datiscetin wurde mit saurem chromsaurem Kali und Schwefelsäure der Destillation 
unterworfen; die übergehende Flüssigkeit enthielt keine öligen Tropfen, hatte aber den Geruch nach salicyliger Säure und färbte sich auf Zusatz von Eisenoxydsalzen purpurroth. Es scheint sich also eine Spur salicyliger Säure gebildet zu haben.

Aus den hier beschriebenen Versuchen scheint mir hervorzugehen, dafs das Datiscin, wie das Salicin, Phloridzin u. a., ein Glucosid ist, und dafs es dem Salicin näher steht, als irgend ein anderes Glucosid, mit Ausnahme des Populins. In der That kenne ich aufser Salicin und Populin kein anderes Glucosid, welches bei dem Erhitzen mit Salpetersäure Nitrosalicylsäure oder gar Pikrinsalpetersäure giebt. Phloridzin und Phloretin geben z. B. nach Aussage verschiedener Beobachter bei der Einwirkung von Salpetersäure nur Oxalsäure. Ich wiederholte den Versuch mit Phloretin und erhielt viel Oxalsäure, während die rückständige Flüssigkeit bei Behandlung mit unterchlorigsaurem Kalk keine Spur Chlorpikrin gab und somit keine Pikrinsalpetersäure enthielt. Quercitrin wurde gleichfalls mit Salpetersäure behandelt und gab, übereinstimmend mit Rigaud's Angabe, nur Oxalsäure. Ich hatte keine Gelegenheil, mit Aesculetin in derselben Richtung Versuche anzustellen, aber diejenigen Chemiker, welche sich mit seiner Untersuchung beschäftigt haben, geben an, dais es nur Oxalsäure liefert.

Ich will diese Mittheilung über Datiscin mil einem Vorschlag, die practische Anwendung dieser Substanz betreffend, schliefsen. Wie bekannt wird der färbende Bestandtheil des Krapps bei dem Kochen mit verdünnter Schwefelsäure zu Zucker und Garancin, einem neuen Farbstoff, welcher für gewisse Zwecke dem in dem Krapp ursprünglich enthaltenen vorzuziehen ist. Hr. Liesching erhielt aus den im Wau und in Quercitronrinde enthaltenen Farbstoffen durch Behandlung mit verdünnter Schwefelsäure färbende Substanzen, 
welche in Wasser nur wenig löslich sind und sich in Beziehung auf färbende Kraft dreimal wirksamer verhalten, als die ursprünglichen Farbstoffe aus denen sie dargestellt waren. Da das Datiscin bei dem Kochen mit verdünnter Schwefelsäure einer ganz ähnlichen Umwandlung unterliegt, indem es sich zu Zucker und Datiscetin spaltet, welches letztere eine viel bedeutendere färbende Kraft als das Datiscin besitzt, so zweifle ich nicht, dafs es für die Anwendung der Datisca cannabina in der Seidefärberei vortheilhaft sein wird, das darin enthaltene Datiscin durch Kochen mit verdünnter Schwefelsäure in Datiscetin umzuwandeln, namentlich da die Operation eine sehr einfache ist und das Datiscetin wegen seiner geringen Löslichkeit in Wasser sehr leicht in verhältnifsmälsig hohem Grad von Reinheit erhalten werden kann.

(Der Schlufs der Abhandlung folgt im nächsten Heft.)

\section{Ueber eine Verbindung des Baryts mit Alkohol; nach M. Berthelot*).}

Eine der von Dumas und Pélig ot entdeckten Verbindung des Baryls wit Holzgeist, $\mathrm{BaO}, \mathrm{C}_{2} \mathrm{H}_{4} \mathrm{O}_{2}$, analoge Verbindung des Baryts mit Alkohol bildet sich nach Berthelot bei Einwirkung von Baryt auf vollkommen wasserfreien Alkohol. Setzt man entwässerten Baryt zu käuflichem s. g. absolutem Alkohol, so zerfällt ersterer; die nach 1 bis 2 Tagen bei Abschlufs der atmosphärischen Feuchtigkeit abfiltrirte Flüssigkeit hält nur etwa $\frac{1}{1}$ бo an Baryt gelöst. Setzt man zu dieser Flüssigkeit wiederum entwässerten Baryt, so zerfält

*) Ann. chim. phys. [3] XLVI, 180. Die Verbindung von Baryt mit Alkohol, in farrenkrautähnlichen Blättern krystallisirt, hatte bereits KubImann erhalten; vgl. diese Annalen XXXIII, 104.

D R. 